\title{
Rosmarinic Acid Intravitreal Implants: A New Therapeutic Approach for Ocular Neovascularization
}

\author{
Authors
}

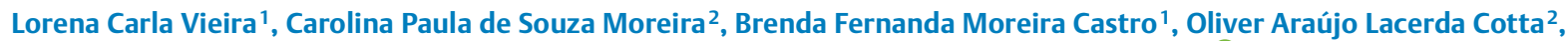
Luciana Maria Silva², Gustavo de Oliveira Fulgêncio ${ }^{1}$, Armando Silva-Cunha ${ }^{1}$, Sílvia L. Fialho ${ }^{2}$

\section{ABSTRACT}

Rosmarinic acid, a plant-derived compound with antiangiogenic activity, can be applied for the treatment of ocular diseases related to neovascularization, such as diabetic retinopathy, macular edema, and age-related macular degeneration. These diseases represent the leading causes of blindness worldwide if they are not properly treated. Intravitreal devices allow for localized drug delivery to the posterior segment, increasing the drug bioavailability and promoting extended release, thus, reducing side effects and enhancing the patient's compliance to the treatment. In this work, rosmarinic acidloaded poly lactic-co-glycolic acid intraocular implants were developed with a view for the treatment of ocular neovascularization. Physical-chemical, biocompatibility, and safety studies of the implants were carried out in vitro and in vivo as well as an evaluation of the antiangiogenic activity in a chorioallantoic membrane assay. Data obtained showed that rosmarinic acid released from the implants was quantified in the vitreous for 6 weeks, while when it was in the solution formulation, after $24 \mathrm{~h}$, no drug was found in the vitreous. The delivery device did not show any sign of toxicity after clinical evaluation and in electroretinographic findings. Histological analysis showed normal eye tissue. Rosmarinic acid released from implants reduced $30 \%$ of new vessel's formation. The intravitreal implant successfully allowed for the prolonged release of rosmarinic acid, was safe to rabbits eyes, and demonstrated activity in vessel reduction, thus demonstrating potential in preventing neovascularization in ophthalmic diseases.

\section{Introduction}

Rosmarinic acid $\left(\mathrm{C}_{18} \mathrm{H}_{16} \mathrm{O}_{8}\right)$ is an ester of $\alpha$-o-caffeoyl-3,4-dihydroxyphenyl lactic acid and is widely found in nature. It mainly belongs to plants in the Boraginaceae and Lamiaceae families and was originally isolated in 1958 from rosemary (Rosmarinus officinalis) $[1,2]$.

Numerous biological activities have been described for rosmarinic acid, such as astringent, antioxidative, anti-inflammatory, antimutagenic, antibacterial, and antiviral [1]. The antioxidant activity of rosmarinic acid is related to its antiangiogenic activity by the inhibition of important steps of angiogenesis, including prolif- eration, migration, and adhesion in a concentration-dependent manner [3-5].

Previous studies have investigated rosmarinic acid therapeutic effects in suppressing retinal and subconjunctival neovascularization, inhibiting pterygium epithelial cells, and preventing cataracts [5-10]. Diabetic retinopathy, macular edema, and age-related macular degeneration are associated with neovascularization and can cause blindness if they are not properly treated [6, 11]. Intravitreal injections are normally used for the treatment of these diseases. However, rosmarinic acid, when administered in the form of aqueous solution, may present low bioavailability, making the use of repeated intravitreal injections necessary, 


$\begin{array}{ll}\text { ABBREVIATIONS } \\ \text { ARPE-19 } & \text { human retinal pigmented epithelial cells } \\ \text { AUC } & \text { area under curve } \\ \text { CAM } & \text { chicken embryo chorioallantoic membrane } \\ \text { C }_{\max } & \text { maximum concentration } \\ \text { DSC } & \text { differential scanning calorimetry } \\ \text { ERG } & \text { electroretinography } \\ \text { FTIR } & \text { Fourier transform infrared spectroscopy } \\ \text { IL-6 } & \text { interleukin 6 } \\ \text { NF-KB } & \text { factor nuclear kappa beta } \\ \text { PLGA } & \text { poly lactic-co-glycolic acid } \\ \text { TGF- } \beta & \text { transforming growth factor beta } \\ \text { T }_{\text {max }} & \text { time to reach the maximum concentration } \\ \text { VEGF } & \text { vascular endothelial growth factor }\end{array}$

which can cause serious complications such as intraocular hemorrhage, retinal detachment, endophthalmitis, and cataracts in addition to great discomfort to patients $[6,12,13]$.

In order to allow the prolonged release of therapeutic levels of drugs in the vitreous, retina, and choroid, increased bioavailability, reduced systemic adverse effects, and intravitreal delivery systems are great choices [14]. They can reduce the complications of intravitreal injections and increase patients' comfort and adherence to treatment $[14,15]$. The use of biodegradable polymeric matrices of PLGA in solid implantable devices have been extensively investigated for ocular administration due to their biocompatibility, predictable kinetics of biodegradation, and mechanical resistance [15-17].

In this work, a biodegradable PLGA implant containing rosmarinic acid was developed and its potential for the prolonged intravitreal release of the drug was evaluated as well as its antiangiogenic activity and biocompatibility in vitro and in vivo.

\section{Results and Discussion}

The treatment of diseases affecting the posterior segment of the eye is limited and challenging once the conventional forms of drug administration fail to provide therapeutic levels of drugs to the vitreous, retina, and choroid [18]. Biodegradable implants are able to release drugs directly to the vitreous and maintain a long-term concentration in the therapeutic range [15].

In order to study the potential to promote a prolonged release of rosmarinic acid, PLGA implants loaded with the drug were developed. They were rod-shaped, homogeneous systems approximately $0.45 \mathrm{~mm}$ in diameter and $6 \mathrm{~mm}$ in length ( $\bullet$ Fig. 1). The mean weight was $1.60 \pm 0.15 \mathrm{mg}$ and they contained approximately $400 \mathrm{\mu g}$ of the drug.

Differential scanning calorimetry was carried out to investigate the drug-polymer interaction after implant preparation. The curve ( $\vee$ Fig. 2 a) of raw PLGA shows an endothermic event at $57.5^{\circ} \mathrm{C}$ corresponding to the glass transition temperature of the polymer [19], and other endothermic events observed between 330 and $365^{\circ} \mathrm{C}$ are attributed to the thermal decomposition of the polymer [20]. Thermal analysis of raw rosmarinic acid

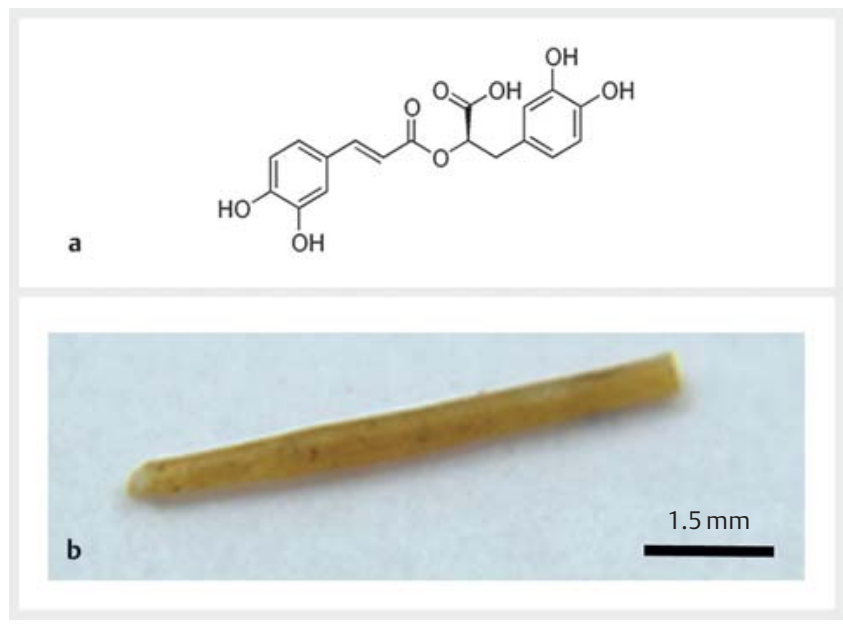

- Fig. 1 a Molecular structure of rosmarinic acid. b Rosmarinic acid/poly lactic-co-glycolic acid implant.

( $\vee$ Fig. 2 b) shows an endothermic event at $65.8^{\circ} \mathrm{C}$, which could be related to the rosmarinic acid glass transition, indicating that part of the drug was in the amorphous state. The drug melting point was observed at $167.3^{\circ} \mathrm{C}$ and an endothermic event starting at $220^{\circ} \mathrm{C}$ indicates rosmarinic acid thermal decomposition [21]. Since no thermal events suggestive of degradation are observed in the temperature range of $70-90^{\circ} \mathrm{C}$, the technique used to mold the implants, the hot molding technique, is appropriate to prepare rosmarinic acid-loaded implants. The curve of the lyophilized mixture of rosmarinic acid and PLGA ( $\vee$ Fig. 2 c) shows endothermic events attributed to PLGA glass transition and decomposition as well as the drug melting point. However, a shift in the PLGA glass transition temperature and rosmarinic acid melting point was observed, probably due to a physical interaction between them [22]. Thus, further analysis by FTIR was performed to investigate this possible interaction.

The FTIR spectrum of raw PLGA 75:25 ( $\bullet$ Fig. 3 a) showed a characteristic band at $1748 \mathrm{~cm}^{-1}$ attributed to $\mathrm{C}=\mathrm{O}$ stretching from the ester groups. Absorption bands at 2947 and $2995 \mathrm{~cm}^{-1}$ corresponding to $\mathrm{C}-\mathrm{H}$ stretching were also identified, as well as bands at the $1460-1000 \mathrm{~cm}^{-1}$ region, characteristics of $\mathrm{C}-\mathrm{O}$ and $\mathrm{O}-\mathrm{H}$ stretching. Similar spectrums for PLGA 75:25 have been reported in the literature $[20,23]$. The rosmarinic acid spectrum ( $\vee$ Fig. 3 b) showed bands at 1607,1515 , and $1464 \mathrm{~cm}^{-1}$, related to $\mathrm{C}-\mathrm{C}$ stretching vibrations in the aromatic ring. $\mathrm{A}$ band at $3165 \mathrm{~cm}^{-1}$, typical of $\mathrm{C}-\mathrm{H}$ stretching in aromatic compounds, was observed. In the same region, bands related to $\mathrm{O}-\mathrm{H}$ stretching from carboxylic acid and a phenol group were also identified. The presence of a phenol group was also evidenced by bands at 1348 and $1180 \mathrm{~cm}^{-1}$ due to $\mathrm{O}-\mathrm{H}$ bending and $\mathrm{C}-\mathrm{O}$ stretching, respectively. Bands at 1724 and $1706 \mathrm{~cm}^{-1}$ are attributed to $\mathrm{C}=\mathrm{O}$ stretching from ester and carboxylic acid groups, respectively. This spectrum is in accordance with the ones described in other studies for raw rosmarinic acid $[21,24]$. The analysis of the solid mixture of PLGA and rosmarinic acid showed that their main groups were preserved ( $\triangleright$ Fig. $\mathbf{3 c}$ ). Similarly, the spectrum of a lyophilized mixture of PLGA and rosmarinic acid ( $\bullet$ Fig. $\mathbf{3 d}$ ) did 


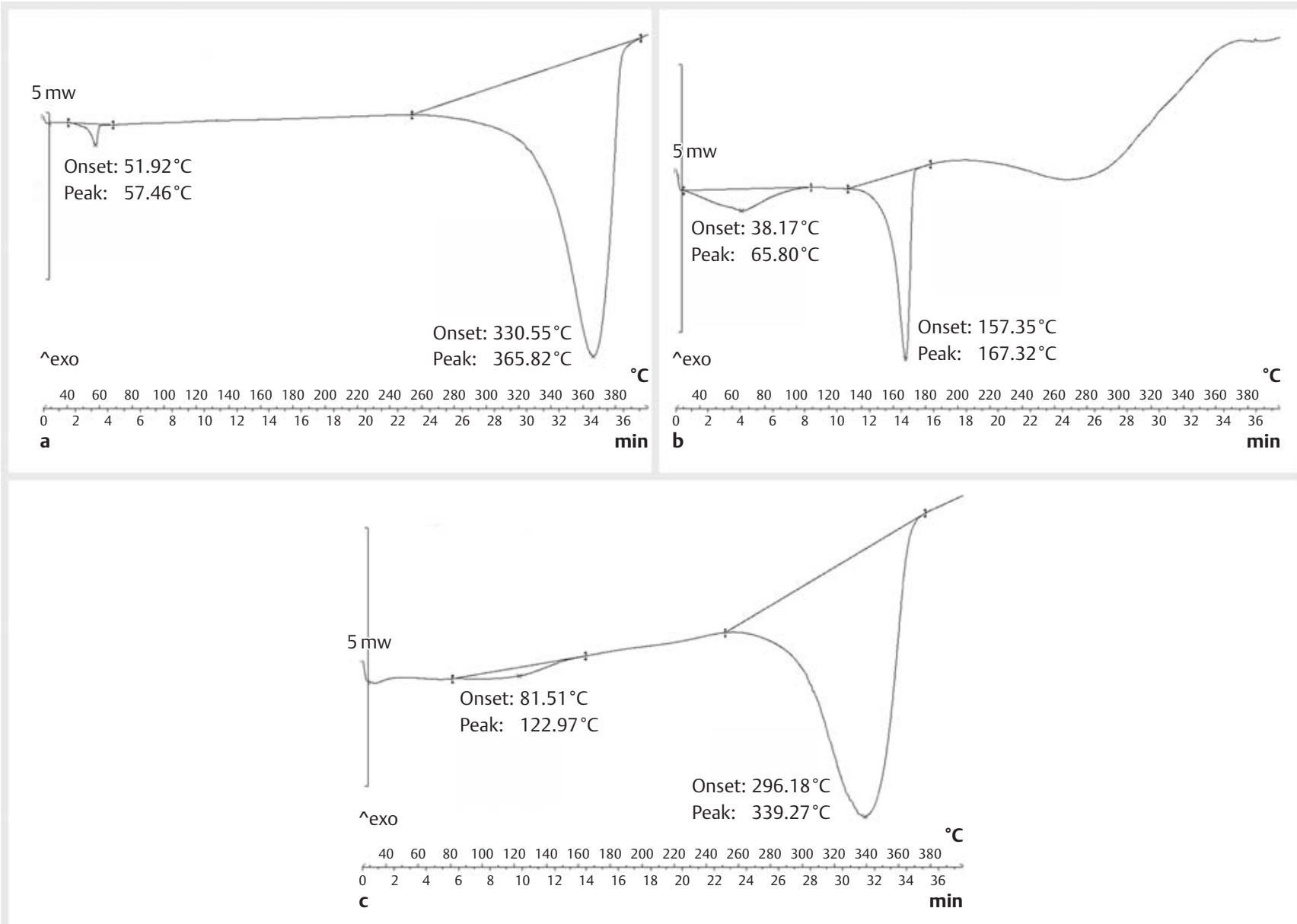

- Fig. 2 Endothermic events observed in differential scanning calorimetry. a DSC curve of PLGA. b DSC curve of RA. c DSC curve of the lyophilized mixture of RA and PLGA. DSC: differential scanning calorimetry, PLGA: poly lactic-co-glycolic acid, RA: rosmarinic acid.

not show modifications in FTIR bands from functional groups present in PLGA and rosmarinic acid. The different shape observed for an $\mathrm{O}-\mathrm{H}$ stretching band at the region of $3451 \mathrm{~cm}^{-1}$ might be due to intermolecular interactions of this group, which superimposed the $\mathrm{C}-\mathrm{H}$ stretching occurring in this region [22, 25]. Both mixtures presented less intense bands for the rosmarinic acid groups, which could be explained by drug dispersion throughout the polymeric matrix. The absence of major changes in the FTIR bands of PLGA and rosmarinic acid suggests there is no chemical interaction between them after lyophilization.

Scanning electronic microscopy photomicrographs showed that rosmarinic acid-loaded implants presented a smooth and homogeneous surface before incubation, without visual pores and channels ( $\bullet$ Fig. 4). After incubation, pores and channels were observed, probably due to polymer degradation that involves breaking ester bonds of the PLGA chain by hydrolytic attack of water molecules of the media [26]. This facilitates drug release by diffusion throughout the polymeric matrix $[27,28]$. Rosmarinic acid is a small compound with slight solubility in water $(1.3 \mathrm{~g} / \mathrm{L}$; $3.6 \times 10^{-3} \mathrm{~mol} / \mathrm{L}$ ). Its small size and linear chain facilitated the passage through the formed porous in PLGA implants during the release study. After 8 days of incubation, more pores and channels were observed ( $\bullet$ Fig. 4 ), which matches with the peak of drug release. The accumulated in vitro release of rosmarinic acid was approximately $92.7 \%$ for 21 days, with $80 \%$ of the drug released within the first 10 days ( $\triangleright$ Fig. 4).

The safety of rosmarinic acid, blank implants, and rosmarinic acid-loaded implants was assessed in vitro in human retinal pigmented epithelial cells. The assay of rosmarinic acid showed that the drug, even at high concentrations, did not affect the viability of the cells. The increase of treatment time did not demonstrate a significant difference in cell viability after 24 and 72 h ( $\bullet$ Fig. 5 a). For blank PLGA implants and those containing $25 \% \mathrm{w} / \mathrm{w}$ of rosmarinic acid, after $72 \mathrm{~h}$ of treatment, the viability of retinal cells was not affected, as observed by the absence of inhibition halos that are caused by the rupture of the cell membrane due to the necrotic process when the material is harmful to the cells ( Fig. 5 b).

The antiangiogenic activity of rosmarinic acid released from the implants was evaluated using the CAM assay, where they were placed over. Treatment with bevacizumab (positive control) at concentrations of 250 and $500 \mu \mathrm{g} / \mathrm{mL}$ significantly reduced the percentage of blood vessels $29.93 \pm 5.41 \%(p<0.0001)$ and $31.51 \pm 5.04 \%(p<0.0001)$, respectively, when compared with 


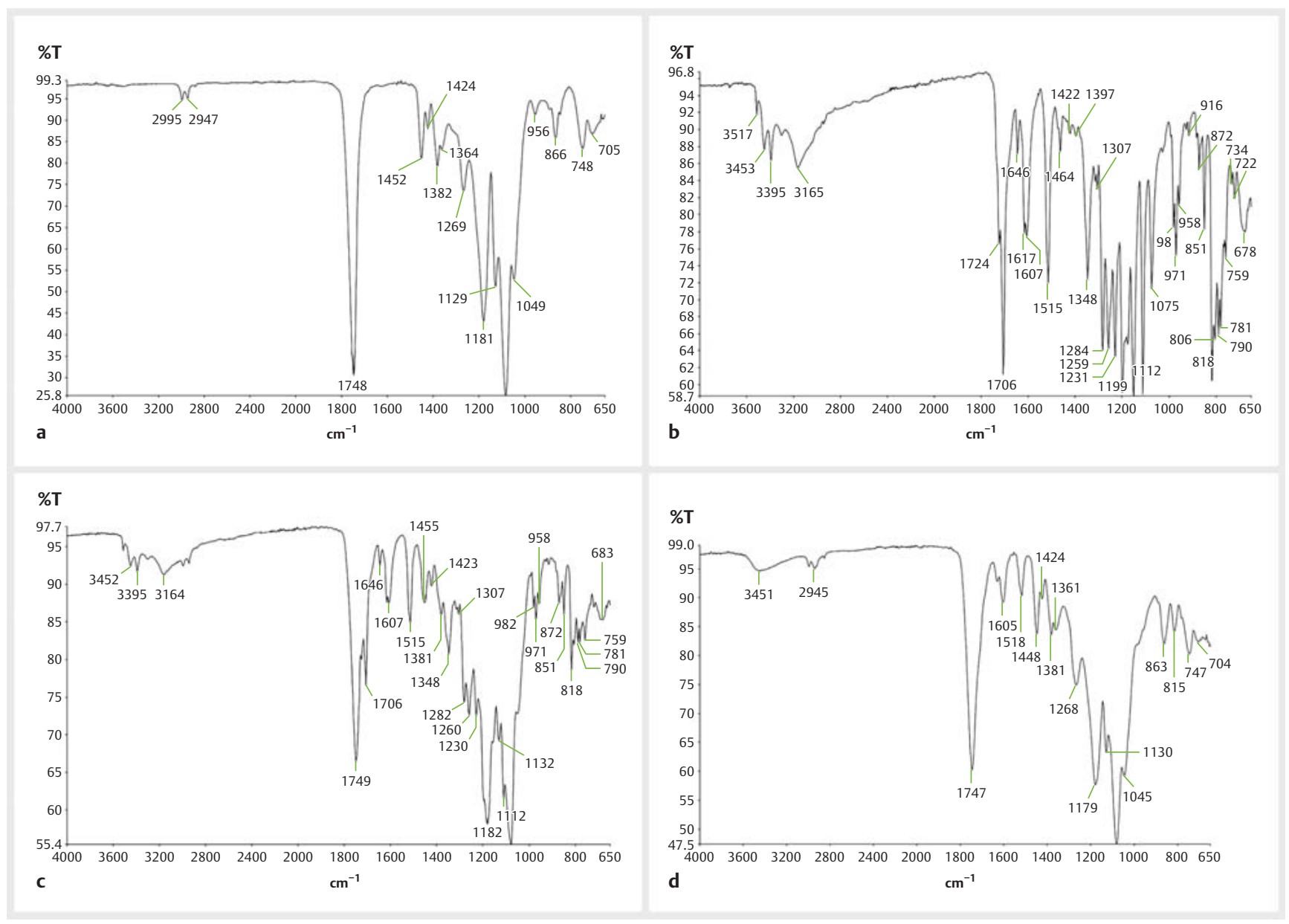

- Fig. 3 FTIR spectrum of (a) raw PLGA 75:25, (b) RA, (c) solid mixture of PLGA and RA, and (d) lyophilized mixture of PLGA and RA. FTIR: Fourier transform infrared spectroscopy; PLGA: poly lactic-co-glycolic acid; RA: Rosmarinic Acid.

the negative control group (treated with PBS). The application of rosmarinic acid solutions at concentrations of 250 and $500 \mu \mathrm{g} / \mathrm{mL}$ on the membrane promoted a significant reduction of $34.58 \pm$ $3.70 \%(p<0.0001)$ and $45.70 \pm 4.76 \%(p<0.0001)$ in blood vessels, respectively, when compared with the negative control group. The percentage of reduction in blood vessels for bevacizumab and rosmarinic acid groups, at the same concentrations, was not significantly different $(p=0.5787$ and $p=0.2475$ for 250 and $500 \mu \mathrm{g} / \mathrm{mL}$, respectively), which was expected ( $\triangleright$ Fig. 6). A significant reduction in blood vessels for the rosmarinic acid-loaded implants group $(71.22 \pm 11.50 \%, p=0.0043)$ was observed when compared with the negative control and blank implants group $(p=0.0087)$. These results suggest that rosmarinic acid was released from the implants at effective concentrations. The CAM assay is an intermediate step between in vitro and in vivo studies and offers advantages to study vascular functions of drugs and formulations. In this study, bevacizumab was used as a positive control since it has well-known antiangiogenic activity and is clinically used in intravitreal injections for the treatment of ocular diseases causing neovascularization [29]. The reduction of vessels with rosmarinic acid alone was similar to bevacizumab and confirms its potential in the treatment of ocular neovascularization, as previ- ously reported [5]. Rosmarinic acid antiangiogenic activity was previously reported to be related to its ability to inhibit proliferation, migration, adhesion, and tube formation of endothelial cells [30]. It has also been reported that rosmarinic acid suppresses the secretion of angiogenic factors such as VEGF, TGF- $\beta$, IL-6, and TNF- $\alpha$, partly via the inhibition of NF- $\kappa$ B p65 [31]. The drug was delivered by the implant in an effective concentration without any sign of inflammation, neovascularization, or vascular lysis.

Intravitreal injection of an aqueous solution of rosmarinic acid $(400 \mu \mathrm{g} / \mathrm{mL})$ in rabbits suggests a low bioavailability of the drug alone, which is better visualized when the AUC values are compared ( $\vee$ Table 1 ). This is probably due to its fast dissolution and consequent elimination from the vitreous. Rosmarinic acid loaded in implants demonstrated an AUC more than 10 times higher than that of the drug in solution. The $C_{\max }$ of rosmarinic acid was observed $1 \mathrm{~h}$ after injection ( $\vee$ Table 1 ) and then the levels started to decrease, reaching $16.23 \pm 5.54 \mu \mathrm{g} / \mathrm{mL}$ at $18 \mathrm{~h}$. After $24 \mathrm{~h}$, only a small amount of rosmarinic acid was detected in the vitreous of the animals, below the quantification limit of the method ( $\vee$ Fig. 7a). Differently, the rosmarinic acid-PLGA implants inserted in the vitreous demonstrated a prolonged release profile of the drug ( $\triangleright$ Fig. 7 b). An initial peak of $20.47 \pm 5.21 \mu \mathrm{g} / \mathrm{mL}$ of 

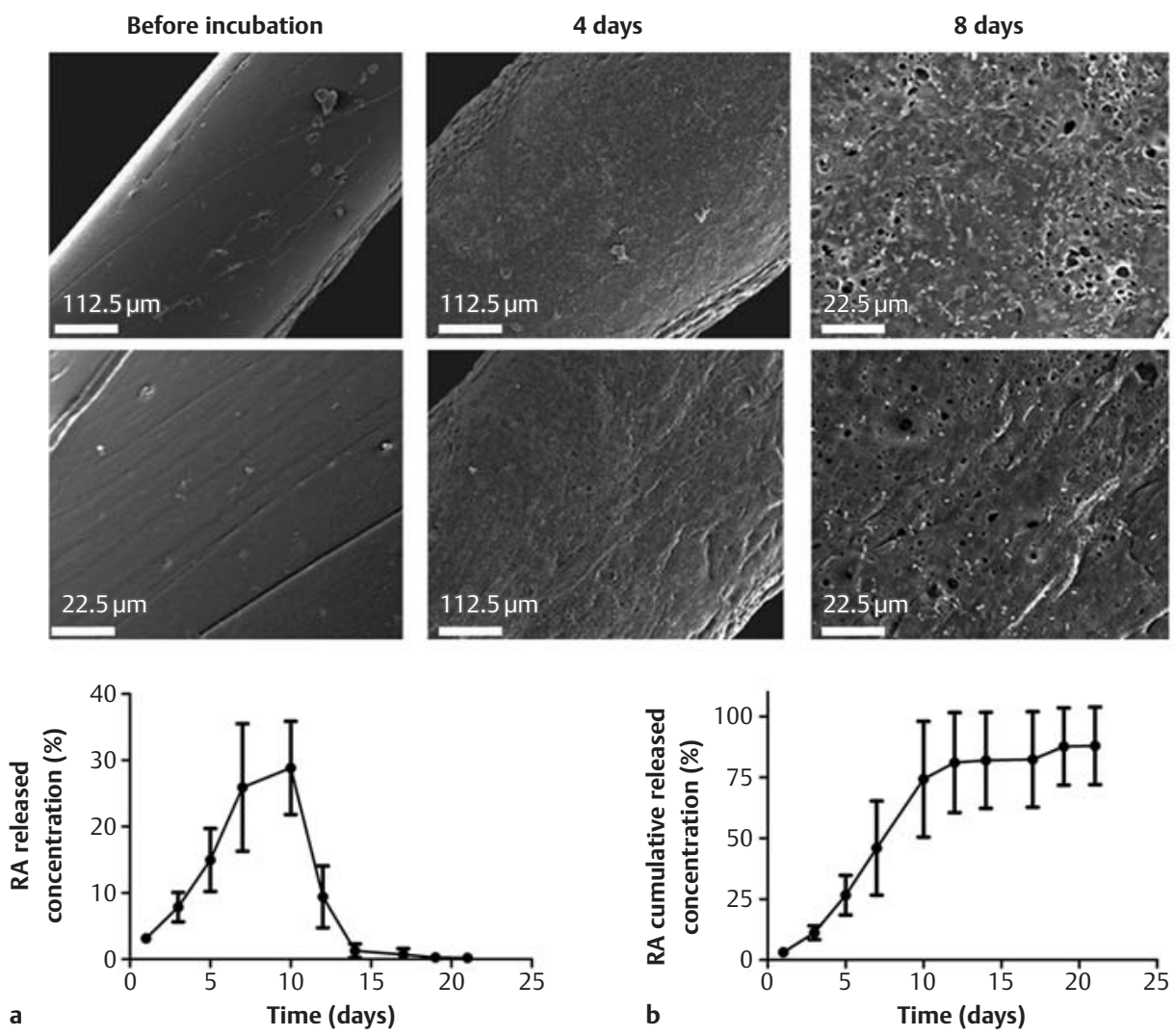

- Fig. 4 In vitro degradation and release profile of rosmarinic acid implants. Top: images of rosmarinic acid/poly lactic-co-glycolic acid implants obtained by scanning electronic microscopy. Bottom: in vitro release profile of rosmarinic acid (RA) from implants during 6 weeks. a \% of RA released in medium. b Cumulative concentration (\%) of RA released in medium (values are shown as the mean $\pm S D, n=6$ ).

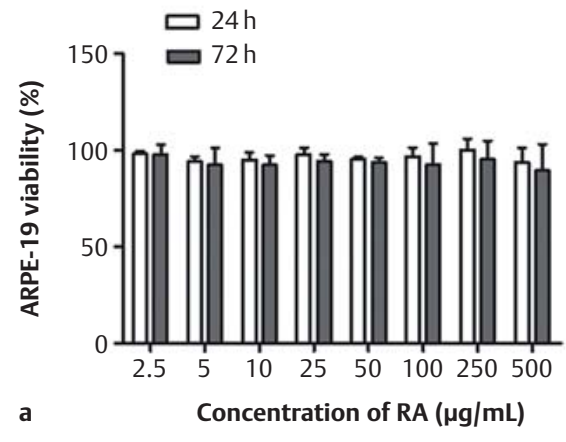

- Fig. 5 Cell viability assay using RA solutions and implants at 24 and $72 \mathrm{~h}$. a RA solution in ARPE-19 (values are shown as the mean $\pm S D, n=3$ ). b Intravitreal implants in ARPE-19 (diffusion method in agar) showing the absence of halos: control (top), PLGA implant (middle), PLGA/RA implant (bottom). ARPE: retinal pigmented epithelial cells, PLGA: poly lactic-co-glycolic acid, RA: rosmarinic acid. 


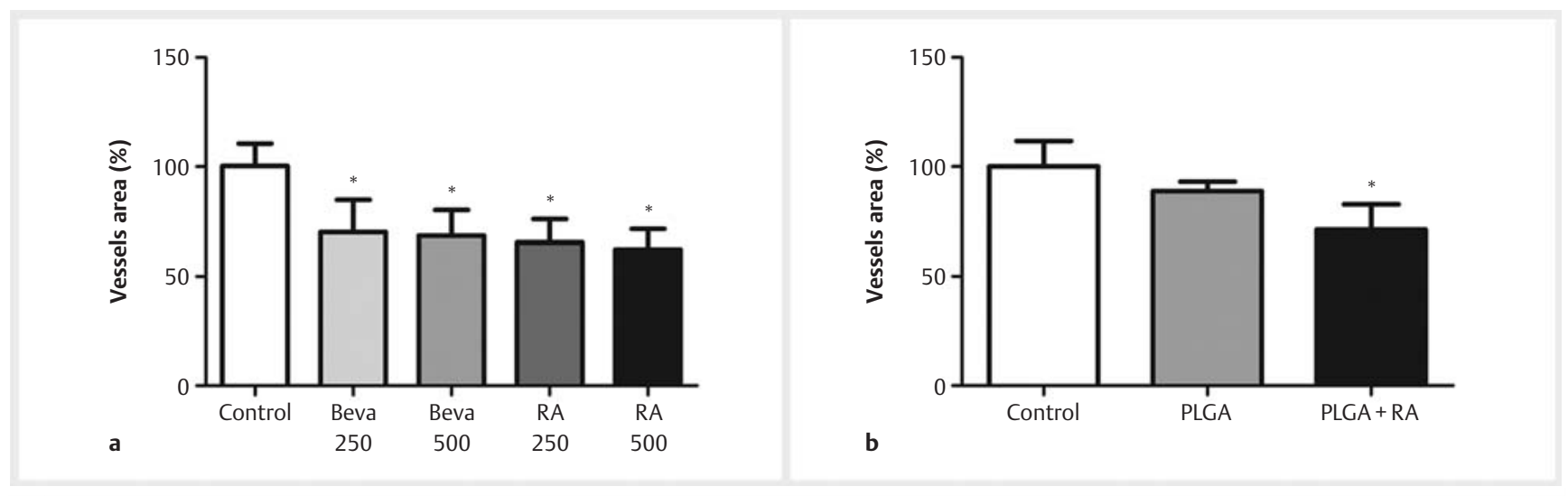

> Fig. 6 Quantification of blood vessels of the chorioallantoic membrane (CAM). a Solutions of rosmarinic acid (RA) at $250 \mu \mathrm{g} / \mathrm{mL}$ (RA 250 ) and $500 \mu \mathrm{g} / \mathrm{mL}$ (RA 500) and bevacizumab at $250 \mu \mathrm{g} / \mathrm{mL}$ (Beva 250) and $500 \mu \mathrm{g} / \mathrm{mL}$ (Beva 500). b Poly lactic-co-glycolic acid (PLGA) and PLGA/RA implants (values are shown as the mean $\pm S D, n=12$ ). To calculate the \% of vessel area of the CAM, the images were converted to grayscale using the software ImageJ and the pixels were quantified. The control group was set to $100 \%$.

rosmarinic acid observed 5 days after insertion is probably due to the drug present on the surface of the implants that is rapidly eliminated from the system to the medium. At 21 days, the maximum concentration of the drug was observed and might be related to the drug being released from the pores and channels formed during the matrix degradation ( $\triangleright$ Table 1 ). After this period, statistical analysis demonstrated that the rosmarinic acid was released from the implants at similar concentrations $(p<0.05)$, which suggests a controlled release profile. The drug could be quantified in vitreous for up to 40 days. Considering the values of $\mathrm{T}_{\max }$ of the rosmarinic acid solution and rosmarinic acidloaded implants, when the drug is incorporated in the delivery system, it is shifted to a later time, demonstrating the prolonged release $(\triangleright$ Table $\mathbf{1})$. Further, when we compare the values of the slope of the log-scale graphs ( $\triangleright$ Fig. 7 c, d), we observed a higher inclination in the curve of the rosmarinic acid solution than that loaded in the implants, which shows that the drug concentration reduces faster in the first. Thus, the intravitreal device developed was able to promote a more prolonged and controlled release of rosmarinic acid in the vitreous of rabbits, suggesting a more efficient treatment, with the possibility of reduced adverse effects and higher adherence of the patient to the treatment.

Although many improvements have been made in the last years in order to enhance ocular bioavailability of drugs, reaching therapeutic doses in the posterior segment is still a challenge, and intraocular diseases are likely to emanate and prevail. The use of intravitreal devices capable of delivering drugs over a longer period of time, such as the polymeric implant developed in this work, is a potential alternative for the treatment of intraocular diseases. They can replace repeated intravitreal injections and reduce their associated adverse effects, increasing the adhesion of the patient to the treatment $[13,31-33]$.

Clinical evaluation of the animals performed weekly after insertion of the rosmarinic acid-loaded implants showed that the devices did not cause any inflammatory reaction, hemorrhage, or retinal detachment as well as retinal edema ( $\bullet$ Fig. 8 ). There was no significant alteration in the intraocular pressure of the animals
- Table 1 Pharmacokinetic parameters of rosmarinic acid in the vitreous after intravitreal administration of the drug in solution and loaded in implants.

\begin{tabular}{|l|l|l|}
\hline Parameters & RA solution & RA implant $^{a}$ \\
\hline AUC & $815.4 \mu \mathrm{g} / \mathrm{mL} \times \mathrm{h}$ & $9609.0 \mu \mathrm{g} / \mathrm{mL} \times \mathrm{h}$ \\
\hline $\mathrm{T}_{\max }$ & $1 \mathrm{~h}$ & $504 \mathrm{~h}(21$ days $)$ \\
\hline $\mathrm{C}_{\max }$ & $204.49 \pm 31.06 \mu \mathrm{g} / \mathrm{mL}$ & $20.48 \pm 6.20 \mu \mathrm{g} / \mathrm{mL}$ \\
\hline
\end{tabular}

${ }^{a}$ Data of implant release profile was transformed from days to hours for the calculation of the implants pharmacokinetic parameters to better compare the difference. AUC: area under curve, $C_{\max }$ : maximum concentration, RA: rosmarinic acid, $T_{\max }$ : time to reach maximum concentration

throughout the experiment ( $\vee$ Fig. 8). In the ocular fundus evaluation, it was possible to visualize the optic disc region without edema and retinal vessel alterations. Conjunctival hyperemia, the presence of cells, and flare in the anterior chamber, cataract, and vitreous haze were also not observed, indicating the absence of toxicity of the implants.

ERG exams were performed to assess the effect of the rosmarinic acid-loaded implants on retinal function. After the electroretinogram recordings in dark-adapted conditions, changes in a- and b-wave amplitudes were analyzed. The results showed no significant change in the amplitudes of a- and b-waves for all stimuli analyzed after 6 weeks of implantation ( $\triangleright$ Fig. 9). ERG is a noninvasive and fundamental examination in ophthalmology for evaluation of retinal diseases since it represents the electrical activity generated by the retina in response to the luminous stimulus [34]. In this study, we analyzed the rod response $\left(0.01 \mathrm{~cd} \mathrm{~s} / \mathrm{m}^{2}\right)$, a- and b-wave combined response $\left(3.0 \mathrm{~cd} \mathrm{~s} / \mathrm{m}^{2}\right)$, and high-intensity response $\left(10.0 \mathrm{~cd} \mathrm{~s} / \mathrm{m}^{2}\right)$. The a-wave (initial negative deflection) is produced by photoreceptors, whereas the subsequent positive deflection, the b-wave, reflects the response generated by other retinal cells, including photoreceptors, bipolar, amacrine, and 


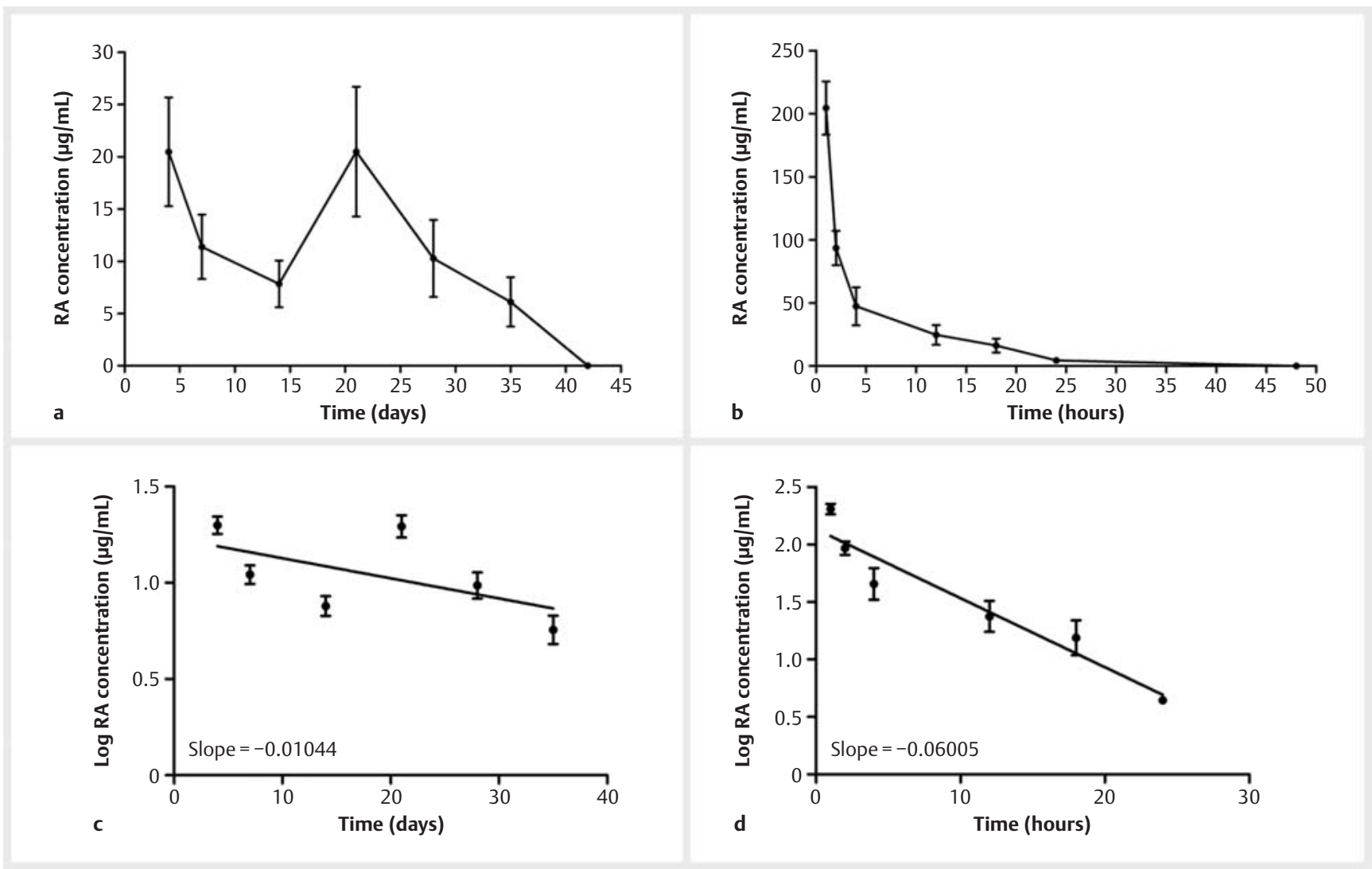

- Fig. 7 In vivo release profile of rosmarinic acid (RA) in solution and incorporated in poly lactic-co-glycolic acid (PLGA) implants with their respective $T_{\max }$ and $C_{\max }$ a $R A$ concentration in the vitreous humor $(\mu \mathrm{g} / \mathrm{mL})$ after 6 weeks of application of the implants. b RA concentration in vitreous humor after intravitreal injection of the drug solution. Log-scale graphs of the concentration of RA released from implants (c) and after RA solution injection ( $d$ ) (the values are shown as the mean $\pm S D, n=6$ ). $T_{\max }$ : time to reach maximum concentration, $C_{\max }$ : maximum concentration.

Muller retinal glial cells [35]. The absence of significant changes in the amplitude of $a$ - and b-waves after insertion of rosmarinic acidloaded implants indicates that the device or the drug released did not promote any harmful toxic effect to the retina, suggesting that it is safe for intravitreal use. Although rosmarinic acid has been previously investigated for the treatment of ocular diseases, studies demonstrating the preservation of retinal function after intravitreal injection of rosmarinic acid have not been reported [5-10].

Histopathological analysis of the retina showed the absence of inflammatory cells and hemorrhages in the areas close to the implant, as well as the integrity of the neuroretina and choroid cells ( $\vee$ Fig. 10). Considering that the architecture of the retina was maintained, it is suggested that there was no damage to photoreceptors caused by the rupture of retinal pigment epithelial cells. Therefore, the retinal layers were not atrophied in the presence of the polymeric implants or the rosmarinic acid released, indicating that there was no toxicity associated.

In this work, we developed a PLGA and rosmarinic acid $(3: 1)$ implant to improve its bioavailability after intravitreal administration. It was verified that the implants were safe to the eye and may have potential to prevent neovascularization in ophthalmic diseases, as they showed antiangiogenic activity ex vivo and were biocompatible and safe to ocular tissues. The prolonged release of rosmarinic acid from the implants shows their suitability for the treatment of chronic ocular diseases that could increase patients' compliance.

\section{Materials and Methods}

\section{Preparation of intravitreal implants}

The implants were prepared by dissolving rosmarinic acid (96\%; Sigma Aldrich) and PLGA 50/50, (PURASORB PDLG 5004, inherent viscosity $0.4 \mathrm{dl} / \mathrm{g}$; Purac Biomaterials) in acetonitrile (HPLC grade; Merck) at a ratio of rosmarinic acid: PLGA of $1: 3$. The solution was lyophilized and the powder obtained was molded into rods at a temperature between $70-90^{\circ} \mathrm{C}$. The implants were prepared so that they contained approximately $400 \mu \mathrm{g}$ of the drug. The mean weight of the implants was calculated from 10 samples randomly selected.

\section{Thermal analysis}

The thermal behavior of the materials used to prepare rosmarinic acid-loaded implants was evaluated by DSC using a DSC50 differential scanning calorimeter (Shimadzu). Samples of $4 \mathrm{mg}$ of raw 

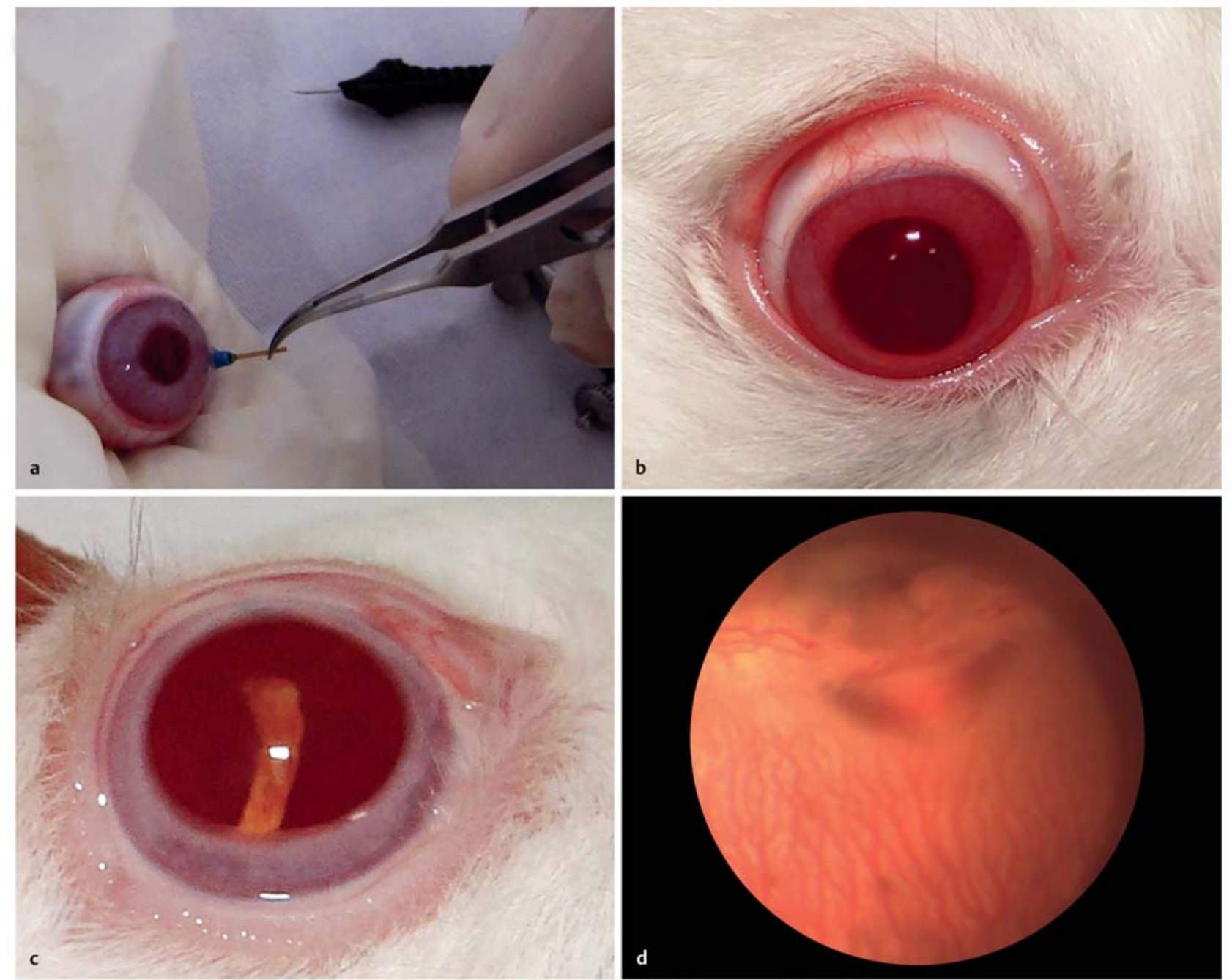

- Fig. 8 a Implant insertion using transscleral trocar canula 25 G. b Site of the implant immediately after insertion, showing the absence of hemorrhage. c Implant into the vitreous cavity. $\mathbf{d}$ Ophthalmoscopy with the normal retina vessels and the optic disc.

rosmarinic acid, raw PLGA, and a lyophilized mixture of rosmarinic acid:PLGA (1:3) were accurately weighted in closed and pierced aluminum pans. The curves were obtained in the temperature range of $25-400^{\circ} \mathrm{C}$ using a $10^{\circ} \mathrm{C} / \mathrm{min}$ heating rate and nitrogen atmosphere.

\section{Fourier transform infrared spectroscopy}

FTIS characterization was performed in a Perkin-Elmer spectrophotometer (model Spectrum 1000; Perkin-Elmer) to investigate the presence of specific chemical groups and interactions among the components. Each spectrum was obtained with a resolution of $4 \mathrm{~cm}^{-1}$ and a spectral range of $4000-650 \mathrm{~cm}^{-1}$. Samples of raw PLGA, raw rosmarinic acid, a solid mixture of raw rosmarinic acid:PLGA $(1: 3)$, and a lyophilized mixture of rosmarinic acid:PLGA $(1: 3)$ were used.

\section{In vitro degradation and release studies}

The in vitro release study was performed in glass vials containing $2 \mathrm{~mL}$ of PBS (pH 7.4), following the sink conditions, with stirring and temperature maintained constant at $30 \mathrm{rpm}$ and $37^{\circ} \mathrm{C}$, respectively. rosmarinic acid-PLGA implants $(n=6)$ were individually placed in the vials. Implants without the drug were also evaluated $(n=6)$. For 21 days, at pre-established time intervals, the medium was completely withdrawn, and the same buffer solution was immediately replaced. The collected samples were analyzed by HPLC using the method described below.

Morphological changes on the surface of blank and rosmarinic acid-loaded implants retrieved from the in vitro release study (days 4 and 8 ) were evaluated by scanning electronic microscopy. After 8 days, the very fragile implants were removed and mounted for analysis. The implants withdrawn from the release media were blot dried and left in a desiccator for $72 \mathrm{~h}$. Then, they were placed in stubs, dried, and gold-coated for $60 \mathrm{~s}$ prior to 

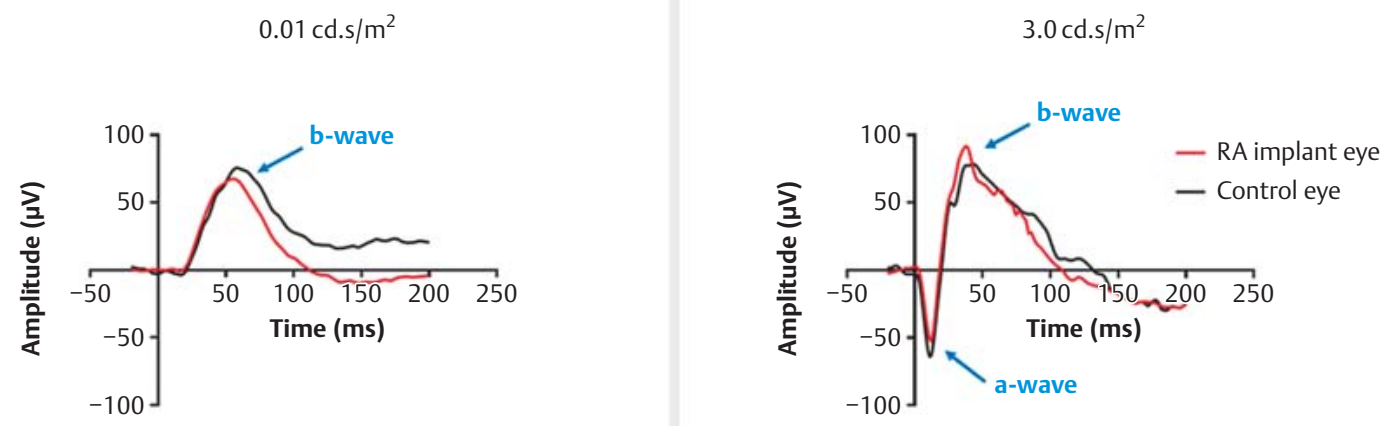

- Fig. 9 Examples of the dark-adapted electroretinography responses of one animal at two luminance stimuli $\left(0.01 \mathrm{and} 3.0 \mathrm{~cd} . \mathrm{s} / \mathrm{m}^{2}\right)$. RA: rosmarinic acid.
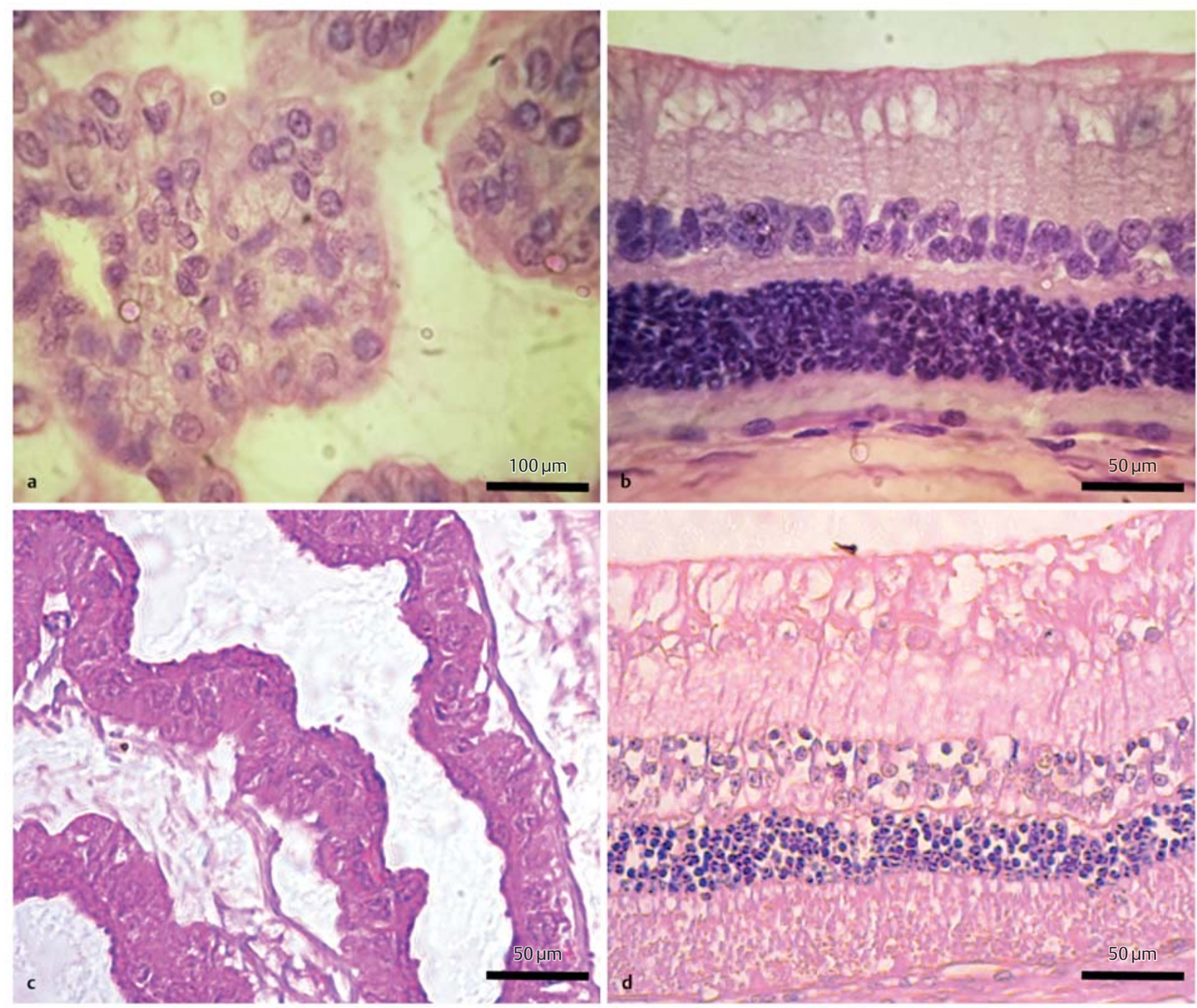

- Fig. 10 Histological sections of the ciliary body and retina 6 weeks after insertion of rosmarinic acid implants (a, b) and before implantation (c, d). Region of the ciliary body with the absence of inflammatory cells (a, c) and retinal images showing the integrity of its layers (b, d). 
analysis. The photomicrographs were obtained at a voltage of $15 \mathrm{Kv}$ in a Zeiss DSM 950 (Carl Zeiss NTS GmbH) microscope.

\section{Quantification of rosmarinic acid HPLC}

The amount of rosmarinic acid in the samples from the in vitro and in vivo release studies was measured by HPLC in the isocratic mode using a Waters apparatus attached to a UV-VIS detector at $331 \mathrm{~nm}$. A reverse-phase 18 column (Lichrosorb; Merck) at $25^{\circ} \mathrm{C}$, a mobile phase composed of a mixture of methanol (HPLC grade; Merck)/water (ultrapure; MilliQ) $50: 50\left(0.1 \% \mathrm{H}_{3} \mathrm{PO}_{4}\right.$ 85\%; SigmaAldrich), and a flow rate of $0.8 \mathrm{~mL} / \mathrm{min}$ were used. The method was selective and specific for the quantification of the drug, as well as linear $\left(r^{2}=0.998\right)$ and accurate, with repeatability and intermediate precision and presenting detection and quantification limits of 0.021 and $0.068 \mu \mathrm{g} / \mathrm{mL}$, respectively.

\section{Safety evaluation in human retinal pigment epithelial cells}

The human retinal pigment epithelial cell line (ARPE-19; CRL2302TM) was obtained from American Type Culture Collection and maintained in a cell bank at Ezequiel Dias Foundation (Brazil) until use.

ARPE-19 cells were used to evaluate the safety of rosmarinic acid alone and incorporated in the implants as well as blank implants. Data were obtained from three independent experiments.

For rosmarinic acid alone, the MTT (98\%; Sigma-Aldrich) assay was used. ARPE-19 cells were seeded in 96 -well plates $\left(1 \times 10^{4}\right.$ cells/well) and treated with increased rosmarinic acid solutions $(2.5$ to $500 \mu \mathrm{g} / \mathrm{mL}$ ). PBS $10 \mathrm{X}$ was used as a death control. After 24 and $72 \mathrm{~h}$, the medium was replaced with MTT solution $(5 \mathrm{mg} /$ $\mathrm{mL}$ ) and fresh medium. After $2 \mathrm{~h}$, the precipitated formazan crystals were solubilized with sodium dodecyl sulfate (99\%; Sigma-Aldrich) and the plates were read at $595 \mathrm{~nm}$ using a microplate reader (ELX 800; BIO-TEK Instruments Inc.) after 18 h. Cell viability was determined as a percentage of control (untreated) viability.

The biocompatibility of the implants was investigated by the diffusion method in agar. ARPE-19 cells were seeded in 6-well plates $\left(2 \times 10^{5}\right.$ cells/well) and incubated for $24 \mathrm{~h}$ in DMEM-F12 (Sigma-Aldrich) medium with $10 \%$ fetal bovine serum. Next, the culture medium was removed and fresh medium containing $10 \%$ fetal bovine serum, $1.5 \%$ agar, and $0.4 \%$ neutral red was added. Rosmarinic acid-PLGA and blank implants were then placed on the prepared gel. Polyethylene was used as a negative control. After $72 \mathrm{~h}$, the samples were evaluated macroscopically by the presence of an inhibition halo.

\section{Antiangiogenic activity}

The antiangiogenic activity of rosmarinic acid released from implants was evaluated by the chicken chorioallantoic membrane assay. Fertilized eggs $(n=12)$ were incubated at $37^{\circ} \mathrm{C}$ and $60 \%$ relative humidity. On the 3 rd day of incubation, a hole of approximately $1.0 \mathrm{~cm}$ in diameter was made in the eggshell. The inner membrane was removed, and the eggs were sealed with adhesive tape. On the 5th day of incubation, $20 \mu \mathrm{L}$ of rosmarinic acid solution $(250$ and $500 \mu \mathrm{g} / \mathrm{mL})$ blank and rosmarinic acid-loaded implants were placed on the egg membrane. Bevacizumab (Avastin, Roche Químicos e Farmacêuticos SA; 250 and $500 \mu \mathrm{g} / \mathrm{mL}$ ) and PBS
$(\mathrm{pH} 7.4)$ were used as positive and negative controls, respectively. On the 7th day of incubation, the membrane was extracted after previous fixation with $3.7 \%$ formaldehyde solution and photographed with a digital camera coupled to a stereomicroscope. The images were analyzed using the software Imagej (version 1.50i; National Institutes of Health). For this, they were converted to grayscale and then the black pixels, which corresponded to the vessels of the chorioallantoic membrane, were quantified. The control group was set to $100 \%$. One-way ANOVA followed by Bonferroni's multiple comparison test was used to compare groups using the software Prism 5.0 (GraphPad Software Inc.).

\section{Animals}

New Zealand white rabbits purchased from Experimental Farm Professor Hélio Barbosa (Igarapé, Brazil), weighing 2.0 to $2.5 \mathrm{~kg}$, kept in individual cages with food and water ad libitum, under controlled temperature and humidity, and a light-dark cycle were used for in vivo experiments. All animals were previously examined and those presenting any ocular abnormality were excluded. The protocol (092/2015) was approved by the Ethics Committee in Animal Experimentation of Ezequiel Dias Foundation, Brazil (approval date June 8, 2016) and all experiments were conducted in accordance with the statement of the Association for Research in Vision and Ophthalmology (ARVO) for the Use of Animals in Ophthalmic and Vision Research and with the EC Directive 86/ 609/EEC for animal experiments.

\section{In vivo release study}

The animals were randomly divided into three groups $(n=6)$ that received rosmarinic acid-loaded implant $(400 \mu \mathrm{g} /$ implant, group I), blank implant (group II), and an intravitreal injection of rosmarinic acid solution $(400 \mu \mathrm{g} / \mathrm{mL}$, group III). The left eye of each animal was used as a control. Rabbits were anesthetized with an intramuscular injection of ketamine hydrochloride $(22 \mathrm{mg} / \mathrm{kg}$; Dopalen, Ceva Saúde Animal Ltda,) and xylazine hydrochloride (3 mg/kg; Anasedan, Ceva Saúde Animal Ltda), and one drop of oxybuprocaine hydrochloride $(4 \mathrm{mg} / \mathrm{mL}$; Oxinest, Cristalia) was topically instilled in the animals' eyes prior to the procedures. Intravitreal injection of rosmarinic acid solution was performed using a 30-gauge needle attached to a 1-mL syringe inserted around $1 \mathrm{~mm}$ posterior to the limbus. After 1, 2, 4, 12, 18, 24, and $48 \mathrm{~h}$, the vitreous was collected and frozen at $-80^{\circ} \mathrm{C}$ until drug analysis.

The implants were inserted in the vitreous cavity using 25gauge transscleral cannula (Alcon) that was placed through the pars plana of the eye in the subtemporal quadrant. After pre-established time intervals $(4,7,14,21,28,35$, and 42 days), vitreous samples were collected and frozen at $-80^{\circ} \mathrm{C}$ until drug analysis.

Before vitreous retrieval, all animals were euthanized with a lethal dose of pentobarbital $100 \mathrm{mg} / \mathrm{kg}$. The released concentrations were compared with each other using the Kruskal-Wallis test followed by Dunn's multiple comparison test $(p<0.05)$. Pharmacokinetic parameters of AUC, $T_{\max }$, and $C_{\max }$ of the groups that received the intravitreal implant or the solution were calculated using the software Prism 5.0 (GraphPad Software Inc.). For better comparison, the time data was transformed from days to hours in the implant group. The results were also plotted as log scale to 
compare the pharmacokinetic pattern between immediate release and loaded formulation, and the respective slopes from the curves were calculated (Prism 5.0; GraphPad Software Inc.).

\section{Clinical evaluation}

Ocular examinations were conducted at baseline and weekly during the 6 weeks following implantation. The presence of any sign of inflammation, bleeding, or the occurrence of retinal detachment was verified by slit lamp biomicroscopy (Kowa SL 15 Slitlamp Biomicroscope) and indirect binocular ophthalmoscopy (Omega 500 Binocular Indirect Ophthalmoscope; Heine Optotechnik). For evaluation of the eye fundus, the Clearview Optical Imaging System (Optibrand) was used.

Intraocular pressure of both eyes of each rabbit was measured weekly using a TonoPen (Tono-Pen XL; Reichert Technologies). Three consecutive measurements were taken for each eye and an average value was used for comparison. To minimize circadian oscillation, the measurements were taken at the same hour in all rabbits. All assessments were randomized by the same veterinary ophthalmologist. The values were compared using the KruskalWallis test followed by Dunn's multiple comparison test $(p<0.05)$.

\section{Functional evaluation}

ERG recordings were carried out in compliance with the International Society for Clinical Electrophysiology guidelines [36] in each eye before inserting the implant and 6 weeks after. Initially, rabbits were allowed to adapt to the dark for at least $3 \mathrm{~h}$ to obtain maximum amplitudes and stable parameters. Afterwards, they were anesthetized, and their pupils were dilated with $0.5 \%$ tropicamide (Mydriacyl; Alcon). The eyes were topically anesthetized with $0.5 \%$ proxymetacaine hydrochloride (Anestalcon; Alcon) immediately before the recordings. Each rabbit was placed facing the light stimulus at a distance of $15 \mathrm{~cm}$. Stainless steel needle reference electrodes were placed subcutaneously in the skin near the lateral canthus of the eyes, and a ground needle electrode (model E5; Grass Technologies) was placed subcutaneously on the back. Bipolar contact lense ERGJet electrodes (Fabrinal SA) were placed on both corneas with $2 \% \mathrm{w} / \mathrm{v}$ methylcellulose. Impedance was set to less than $5 \mathrm{k} \Omega$ at $25 \mathrm{~Hz}$ in each electrode. A white light (6500 K) stimulus was generated by a Ganzfeld LED stimulator (ColorDome desktop Ganzfeld; Diagnosys LLC). The intensity of the light stimulus, with a duration of $4 \mathrm{~ms}$, in a scotopic protocol was recorded according to a modified protocol and reported in the following sequence: rod $\left(0.01 \mathrm{~cd} . \mathrm{s} / \mathrm{m}^{2}\right)$ and combined response $\left(3 \mathrm{~cd} . \mathrm{s} / \mathrm{m}^{2}\right)$, with 30 s inter-stimulus intervals.

Responses were amplified (band-pass filter: $0.3-300 \mathrm{~Hz}$ ) and stored for off-line analysis using Espion (Diagnosys LLC) after averaging 6-40 individual measurements at each step depending on the signal/noise ratio.

\section{Histopathological evaluation}

After ERG analysis, the animals were euthanized with a lethal dose of pentobarbital and the eyes were immediately enucleated and fixed for $48 \mathrm{~h}$ in Davidson's fixative solution: $2 \%$ formaldehyde (37\%; Sigma-Aldrich), 35\% ethanol (absolute; Merck), 10\% glacial acetic acid (99\%, Sigma-Aldrich), and 53\% water (ultrapure; Milli-
Q). Next, tissue samples were dehydrated with increasing concentrations of ethanol, diaphanized in xylene (99\%; Sigma-Aldrich), and embedded in paraffin. Sections of $5 \mu \mathrm{m}$ thickness were cut and stained with hematoxylin and eosin (Sigma-Aldrich). The morphology of the retina, presence of inflammatory cell infiltration, and hemorrhages in the ocular tissues were evaluated by light microscopy.

\section{Contributors' Statement}

Conception and design of the work: A. Silva-Cunha, S.L. Fialho; revision of the manuscript: A. Silva-Cunha, S. L. Fialho, C.P.S. Moreira, B. F. M. Castro, O. A. L. Cotta, G. O. Fulgencio, L. M. Silva; analysis and interpretation of the data: A. Silva-Cunha, L.C. Vieira, C.P.S. Moreira, B. F. M. Castro, O. A. L. Cotta, G. O. Fulgencio, L. M. Silva; resources: A. Silva-Cunha, L. M. Silva; data collection: L.C. Vieira, C.P.S. Moreira, B.F.M. Castro, O.A.L. Cotta, G.O. Fulgencio; drafting the manuscript: S.L. Fialho, L.C. Vieira, C.P.S. Moreira, B. F. M. Castro, O. A. L. Cotta, G. O. Fulgencio, L. M. Silva; funding acquisition: S. L. Fialho.

\section{Acknowledgements}

The authors thank Fundação de Amparo à Pesquisa do Estado de Minas Gerais (FAPEMIG, Brazil), Conselho Nacional de Desenvolvimento Científico e Tecnológico (CNPq, Brazil), and Coordenação de Aperfeiçoamento de Pessoal de Nível Superior (CAPES/MEC, Brazil) for financial support.

Conflict of Interest

The authors declare that they have no conflict of interest.

References

[1] Petersena M, Simmonds MS]. Rosmarinic acid. Phytochemistry 2003; 62: $121-125$

[2] Amoah SKS, Sandjo LP, Kratz JM, Biavatti MW. Rosmarinic acid - pharmaceutical and clinical aspects. Planta Med 2016; 82: 388-406

[3] Silva SB, Ferreira D, Pintado M, Sarmento B. Chitosan-based nanoparticles for rosmarinic acid ocular delivery - in vitro tests. Int J Biol Macromol 2016; 84: 112-120

[4] Nunes S, Madureira R, Campos D, Sarmento B, Gomes AM, Pintado M, Reis F. Therapeutic and nutraceutical potential of rosmarinic acid - cytoprotective properties and pharmacokinetic profile. Crit Rev Food Sci Nutr 2017; 57: 1799-1806

[5] Ferreira JLM, Chahud F, Ramalho LN, Modulo CM, Vieira LC, Reinach PS, Rodrigues MLV, Silva-Cunha A, Paula JS. Rosmarinic acid suppresses subconjunctival neovascularization in experimental glaucoma surgery. Curr Eye Res 2015; 40: 1134-1140

[6] Kim JH, Lee BJ, Kim JH, Yu YS, Kim MY, Kim KW. Rosmarinic acid suppresses retinal neovascularization via cell cycle arrest with increase of p21WAF1 expression. Eur J Pharmacol 2009; 615: 150-154

[7] Chen YY, Tsai CF, Tsai MC, Hsu YW, Lu FJ. Inhibitory effects of rosmarinic acid on pterygium epithelial cells through redox imbalance and induction of extrinsic and intrinsic apoptosis. Exp Eye Res 2017; 160: 96-105

[8] Chemerovski-Glikman M, Mimouni M, Dagan Y, Haj E, Vainer I, Allon R, Blumenthal EZ, Adler-Abramovich L, Segal D, Gazit E, Zayit-Soudry S. Rosmarinic acid restores complete transparency of sonicated human cataract ex vivo and delays cataract formation in vivo. Sci Rep 2018; 8: 9341 
[9] Zych M, Wojnar W, Dudek S, Kaczmarczyk-Sedlak I. Rosmarinic and sinapic acids may increase the content of reduced glutathione in the lenses of estrogen-deficient rats. Nutrients 2019; 11: 803

[10] Tsai CF, Wu JY, Hsu YW. Protective effects of Rosmarinic acid against selenite-induced cataract and oxidative damage in rats. Int J Med Sci 2019; 16: 729-740

[11] Zhou YD, Yoshida S, Peng YQ, Kobayashi Y, Zhang LS, Tang LS. Diverse roles of macrophages in intraocular neovascular diseases: a review. Int J Ophthalmol 2017; 10: 1902-1908

[12] Gariano RF, Gardner TW. Retinal angiogenesis in development and disease. Nature 2005; 438: 960-966

[13] Bisht R, Mandal A, Jaiswal JK, Rupentha ID. Nanocarrier mediated retinal drug delivery: overcoming ocular barriers to treat posterior eye diseases. WIREs Nanomed Nanobiotechno 2017; 10: 1-21

[14] Lee SS, Hughes P, Ross AD, Robinson MR. Biodegradable implants for sustained drug release in the eye. Pharm Res 2010; 27: 2043-2053

[15] Costa BL, Machado RR, Paiva MRB, Serakides R, Coelho MM, Silva-Cunha A, Fialho SL. Sirolimus-loaded biodegradable implants induce long lasting anti-inflammatory and antiangiogenic effects. J Drug Deliv Sci Technol 2018; 44: 373-379

[16] Cunha RB, Siqueira RC, Messias A, Scott IU, Fialho SL, Cunha-Junior ADS, Jorge R. Safety and feasibility of a novel 25-gauge biodegradable implant of dexamethasone for treatment of macular edema associates with retinal vein occlusion. Retin Cases Brief Rep 2018; 12: 50-58

[17] Rowe-Rendleman CL, Durazo SA, Kompella UB, Rittenhouse KD, Di Polo A, Weiner AL, Grossniklaus HE, Naash MI, Lewin AS, Horsager A, Edelhauser HF. Drug and gene delivery to the back of the eye: from bench to bedside. Invest Ophthalmol Vis Sci 2014; 55: 2714-2730

[18] Tsai CH, Wang PY, Lin IC, Huang H, Liu GS, Tseng CL. Ocular drug delivery: role of degradable polymeric nanocarriers for ophthalmic application. Int J Mol Sci 2018; 19: 2830-2850

[19] Fialho SL, Rego MGB, Cardillo JA, Siqueira RC, Jorge R, Silva-Cunha A. Biodegradable implants for intraocular drug delivery. Arq Bras Oftalmol 2003; 66: 891-896

[20] Soares DCF, Oliveira DCP, Barcelos LS, Barbosa AS, Vieira LC, Townsend DM, Rubello D, Barros ALB, Duarte LP, Silva-Cunha A. Antiangiogenic activity of PLGA-Lupeol implants for potential intravitreal applications. Biomed Pharmacother 2017; 92: 394-402

[21] Veras KS, Fachel FNS, Delagustin MG, Teixeira HF, Barcellos T, Henriques AT, Bassani VL, Koester LS. Complexation of rosmarinic acid with hydroxypropyl- $\beta$-cyclodextrin and methyl- $\beta$-cyclodextrin: Formation of 2: 1 complexes with improved antioxidant activity. J Mol Struct 2019; 1195: 582-590

[22] Blasi P, Schoubben A, Giovagnoli S, Perioli L, Ricci M, Rossi C. Ketoprofen poly(lactide-co-glycolide) physical Interaction. AAPS PharmSciTech 2007; 8: E1-E8

[23] Yang J, Lee CH, Park J, Seo S, Lim EK, Song Y], Suh JS, Yoon HG, Huh YM, Haam S. Antibody conjugated magnetic PLGA nanoparticles for diagnosis and treatment of breast cancer. J Mater Chem 2007; 17: 2695-2699
[24] Stehfest K, Boese M, Kerns G, Piry A, Wilhelm C. Fourier transform infrared spectroscopy as a new tool to determine rosmarinic acid in situ. J Plant Physiol 2004; 161: 151-156

[25] Costa HS, Mansur AAP, Pereira MM, Mansur HS. Engineered hybrid scaffolds of poly(vinyl alcohol)/bioactive glass for potential bone engineering applications: synthesis, characterization, cytocompatibility, and degradation. J Nanomater 2012; 2012: 1-16

[26] Chereddy KK, Payen VL, Préat V. PLGA polymer: From a classic drug carrier to a novel therapeutic activity contributor. J Control Release 2018; 289: 10-13

[27] Fernandes-Cunha GM, Rezende CM, Mussel WN, da Silva GR, Elionai CDL, Yoshida MI, Fialho SL, Goes AM, Gomes DA, Vitor RWA, Silva-Cunha A. Anti-Toxoplasma activity and impact evaluation of lyophilization, hot molding process, and gamma-irradiation techniques on CLH-PLGA intravitreal implants. J Mater Sci Mater Med 2016; 27: 10

[28] Fialho SL, Rêgo MB, Siqueira RC, Jorge R, Haddad A, Rodrigues AL jr., Maia-Filho A, Silva-Cunha A. Safety and pharmacokinetics of an intravitreal biodegradable implant of dexamethasone acetate in rabbit eyes. Curr Eye Res 2006; 31: 525-534

[29] Bakri S], Thorne JE, Ho AC, Ehlers JP, Schoenberger SD, Yeh S, Kim S]. Safety and efficacy of anti-vascular endothelial growth factor therapies for neovascular age-related macular degeneration. Ophthalmology 2018; 126: 55-63

[30] Kunou N, Ogura Y, Yasukawa T, Kimura H, Miyamoto H, Honda Y, Ikada Y. Long-term sustained release of ganciclovir from biodegradable scleral implant for the treatment of cytomegalovirus retinitis. J Control Release 2000; 68: 263-271

[31] Cao W, Hu C, Wu L, Xu L, jiang W. Rosmarinic acid inhibits inflammation and angiogenesis of hepatocellular carcinoma by suppression of NF- $\mathrm{BB}$ signaling in H22 tumor-bearing mice. J Pharmacol Sci 2016; 132: 131137

[32] Huang SS, Zheng RL. Rosmarinic acid inhibits angiogenesis and its mechanism of action in vitro. Cancer Lett 2006; 239: 271-280

[33] Cabrera FJ, Wang DC, Reddy K, Achary G, Shin CS. Challenges and opportunities for drug delivery to the posterior of the eye. Drug Discov Today 2019; 24: 1679-1684

[34] Quintana MLQ, Benedetto MM, Maldonado AC, Payer EV, Contin MA. Electroretinography: a biopotential to assess the function/dysfunction of the retina. J Phys Conf Ser 2016; 705: 012053

[35] Chen J, Caspi RR. Clinical and functional Evaluation of ocular inflammatory Disease using the Model of experimental autoimmune Uveitis. In: Boyd A, ed. Immunological Tolerance Methods in molecular Biology. New York: Humana Press; 2019: 211-227

[36] Marmor MF, Fulton AB, Holder GE, Miyake Y, Brigell M, Bach M. ISCEV Standard for full-field clinical electroretinography (2008 update). Doc Ophthalmol 2009; 118: 69-77 